\title{
A RARE CASE OF MEN 4 PRESENTING WITH HYPERCALCAEMIA IN A PATIENT WITH MICROPROLACTINOMA 6 YEARS AFTER THE DIAGNOSIS
}

Kleopatra Alexiadou ${ }^{1}$, Devasenan Devendra ${ }^{1}$, Thomas Galliford ${ }^{1}$, Martina Owens ${ }^{2}$, Anne-Marie Bussell ${ }^{2}$, Nizar Damani ${ }^{1}$, Arla Ogilvie ${ }^{1}$.

${ }^{1}$ Department of Diabetes and Endocrinology, West Hertfordshire NHS Trust, Watford, UK

2 Molecular Genetics Laboratory, Royal Devon and Exeter NHS Trust, Exeter, UK

\section{Background}

Multiple Endocrine Neoplasia is characterised by the occurrence of tumours involving two or more endocrine glands within a single patient. MEN are autosomal dominant disorders.

Four forms have been described: MEN 1 due to menin mutations, MEN2 (previously MEN2A) due to mutations of a tyrosine kinase receptor encoded by the rearranged during transfection (RET) protoncogene, MEN3 (previously MEN2B) due to RET mutations and MEN4 due to cyclin-dependent kinase inhibitor (CDNK1B) mutations.

Each MEN is associated with the occurrence of specific tumours. MEN4 is characterized by the occurrence of parathyroid and anterior pituitary tumours in possible association with tumours of the adrenals, kidneys, and reproductive organs.

\section{Case Presentation}

We are presenting the case of a 31 year old female referred to our Endocrine Clinic by the Fertility Clinic with hyperprolactinaemia (PRL:1158 mu/L) in March 2010. Her MRI pituitary revealed a microprolactinoma and was started on cabergoline with good response (PRL: $156 \mathrm{mu} / \mathrm{L}$ ). In August 2016, she came back in Clinic for her routine follow up.

\section{Investigations}

Her prolactin levels remained normal but hypercalcaemia (CorrCa: 2.70 $\mathrm{mmol} / \mathrm{L}$ ) was noted. Her PTH was subsequently tested and found to be elevated $(9.9 \mathrm{pmol} / \mathrm{L})$ and her Vitamin D levels were normal $(79 \mathrm{nmol} / \mathrm{L})$. The results were in keeping with primary hyperparathyroidism.

MIBI and US parathyroid scans didn't reveal any distinct adenoma. The suspicion of MEN was raised and she was referred for genetic testing. Mutation analysis of the AIP, CDKN1B, MEN 1 and RET genes was requested.

The patient was found to be heterozygous for a novel CDKN1B frameshift mutation consistent with a diagnosis of MEN 4.
Follow up

Each of the patient's offspring is at $50 \%$ risk of inheriting this mutation and developing MEN 4. She has an adopted daughter and a sister who has 3 children. Enquiring more into her family history, it transpired that her father died with a diagnosis of duodenal tumours and somatostatinoma.

She was referred to the Clinical Geneticist, a fasting gut hormone profile was performed and a CT chest/abdomen/pelvis was arranged after discussion at a multidisciplinary meeting with the Radiology Consultant in order to exclude the coexistence of adrenal, renal or ovarian tumours. The fasting gut hormone profile was normal. The CT report raised the possibility of a lower uterine lesion which was not confirmed by the subsequent TV ultrasound scan.

\begin{tabular}{|l|c|c|c|}
\hline & July 2014 & August 2016 & January 2017 \\
\hline Corr Ca (mmol/L) & 2.48 & 2.71 & 2.74 \\
\hline PTH (pmol/L) & - & 9.9 & 11.9 \\
\hline Vitamin D (nmol/L) & 70 & 79 & - \\
\hline
\end{tabular}

\begin{tabular}{l|l}
\hline Genetic testing & April 2017 \\
\hline Novel CDKN1B frameshift mutation \\
consistent with a diagnosis of MEN 4
\end{tabular}

\begin{tabular}{ll} 
& June 2017 \\
\hline CT Chest/Abdomen/Pelvis & ? Lower uterine lesion \\
TV Ultrasound scan & Normal
\end{tabular}

\section{Conclusion}

We are presenting a rare case of MEN 4 presenting with hypercalcaemia in a patient with known microprolactinoma 6 years after the initial diagnosis. This case illustrates the required level of clinical suspicion when encountering cases of young patients with more than one endocrinopathies in order to refer the appropriate cases for genetic testing. It also underlines that the management of these cases requires multidisciplinary approach and input from various specialities (endocrinologists, clinical geneticists, radiologists, oncologists).

\section{References}

1. Schernthaner-Reiter MH, Trivellin G, Stratakis CA. MEN1, MEN4, and Carney Complex: Pathology and Molecular Genetics. Neuroendocrinology 2016;103(1):18-31.

2. Thakker RV. Multiple endocrine neoplasia type 1 (MEN 1) and type 4 (MEN 4). Mol Cell Endocrinol 2014 Apr 5;386(1-2):2-15.

3. Lee M, Peleggata NS. Multiple endocrine neoplasia type 4. Front Horm Res 2013;41:63-78.

4. Norton JA, Krampitz G, Jensen RT. Multiple Endocrine Neoplasia: Genetics and Clinical Management. Surg Oncol Clin N Am 2015 Oct;24(4):795-832. 